\title{
Effects of virtual reality-based core stabilization exercise on upper extremity function, postural control, and depression in persons with stroke
}

\author{
Jee-Won Kim ${ }^{\mathrm{a}(\mathbb{1})}$, Jung-Hee $\mathrm{Kim}^{\mathrm{b}(}{ }^{(1)}$, Byoung-Hee Lee ${ }^{\mathrm{c}(\mathbb{1})}$ \\ ${ }^{a}$ Department of Physical Therapy, Sahmyook Medical Center, Seoul, Republic of Korea \\ ${ }^{\mathrm{b}}$ Department of Physical Therapy, Andong Science College, Andong, Republic of Korea \\ 'Department of Physical Therapy, College of Health Science and Social Welfare, Sahmyook University, Seoul, Republic of Korea
}

Objective: The purpose of this study was to evaluate the effect of virtual reality (VR)-based core stabilization exercise on upper extremity function, postural control, and depression among persons with stroke with hemiplegia.

Design: Randomized controlled trial.

Methods: This study was conducted with the inclusion of 24 participants and were randomly assigned to either the VR-based trunk stability exercise group $(\mathrm{n}=12)$ or control group $(\mathrm{n}=12)$. The VR-based trunk stability exercise group performed core stabilization exercises in a VR environment for 30 minutes. Meanwhile, the control group conducted general core stabilization exercises for 30 minutes. The participants trained 3 times a week for 4 weeks. The manual functional test (MFT), Box and Block Test (BBT), Berg Balance Scale (BBS), Trunk Impairment Scale (TIS), the Geriatric Depression Scale (GDS) were used to assess all participants before and after the intervention.

Results: The VR-based core stabilization exercise group had a significant improvement in upper extremity function (MFT, BBT) and postural control (BBS) compared with the control group $(p<0.05)$. The VR-based core stabilization exercise showed a significant difference after intervention in the TIS and GDS scores $(p<0.05)$, but they did not significantly differ between the two groups.

Conclusions: The result showed that VR-based core stabilization exercise can be effective in improving upper extremity function and postural control among patients with stroke more than the sole application of general physical therapy.

Key Words: Core stabilization, Postural balance, Stroke, Upper extremity, Virtual rehabilitation

\section{Introduction}

After a stroke, body movements and balance abilities are reduced due to abnormal muscle activity and asymmetric posture. Therefore, an individual experiences difficulty in performing complicated tasks while walking or standing. In addition, postural asymmetry impairs the perception of space, reduces the separation of the trunk and limbs, decreases the back and forth movement of the pelvis during weight transfer, and causes difficulties in maintaining spine alignment and equilibrium response [1,2].

Most patients with stroke present with hemiplegia due to sagittal plane displacement of the center of mass, asymmetry in the left and right sides of the body, and concentration and distribution of approximately $61 \%-80 \%$ of the total body weight in the tendon of the limbs [3]. Therefore, improving the alignment of the trunk must be prioritized to achieve better posture control and functional movement associated with activities of daily living. In addition, pelvis and trunk stability is required to effectively mobilize the upper

Received: 3 July, 2020 Revised: 10 August, 2020 Accepted: 20 August, 2020

Corresponding author: Byoung-Hee Lee (ORCID https://orcid.org/0000-0001-9766-6068)

Department of Physical Therapy, College of Health Science and Social Welfare, Sahmyook University, 815 Hwarang-ro, Nowon-gu, Seoul 01795 , Republic of Korea Tel: 82-2-3399-1634 Fax: 82-2-3399-1639 E-mail: 3679@syu.ac.kr

(c) This is an Open-Access article distributed under the terms of the Creative Commons Attribution Non-Commercial License (http://creativecommons.org/licenses/ by-nc/4.0) which permits unrestricted non-commercial use, distribution, and reproduction in any medium, provided the original work is properly cited.

Copyright $@ 2020$ Korean Academy of Physical Therapy Rehabilitation Science 
limbs [4].

In recent years, several studies have emphasized the use of interventions that are actively performed to promote reconstruction and neurodegeneration of intensive, repetitive, reality-related, interventional parts. In relation to this context, interventions involving activities in a virtual space has been proposed [5]. The virtual reality (VR)-based training, which is implemented by reflecting an actual VR space, is used to improve balance and gait, upper limb function, and quality of life in stroke survivors. Lee et al. [6] confirmed the improvement of upper limb function and trunk stability by applying a VR program based on a canoe game three times a week for 4 weeks for 10 participants with stroke. In addition, Cho et al. [7] confirmed a significant difference in Berg balance scale (BBS) and timed up and go test (TUG) as a result of applying a video game system in combination with general physical therapy 3 times a week for 6 weeks for the purpose of improving dynamic balance in individuals with chronic stroke [6-8]. Augmented reality therapy may increase motor function [9] and cognitive ability in elderly individuals [10].

However, there are little studies about the use of the VR-based core stabilization exercise program in stroke survivors, and a therapeutic intervention that can be used in rehabilitation is challenging to establish. Thus, various programs that can supplement it must be developed. The current study aimed to investigate the effect of an augmented real- ity-based core stabilization exercise program on upper extremity function, postural control, and depression among patients with stroke.

\section{Methods}

\section{Participants}

Twenty-four patients who presented with stroke 6 months prior to the study were included. All participants completely understood the content of the study and agreed to participate.

The inclusion criteria for this study were as follows: individuals without musculoskeletal or cardiovascular disease, those with a Korean Mini-Mental State Examination score of 24 or higher, those without visual impairment and visual field defects [11]; and those who could understand and carry out instructions.

The present study was approved by the Institutional Review Board of Sahmyook University (IRB No. 2-104078 1-AB-N-01-2017022HR) and the objective of the study and its requirements were explained to the subjects, and all participants provided written consent in accordance with the ethical principles of the Declaration of Helsinki.

\section{Procedures}

Twenty-four participants were randomly divided into the VR-based core stabilization exercise group $(n=12)$ and the control group $(n=12)$. The participants in both groups re-

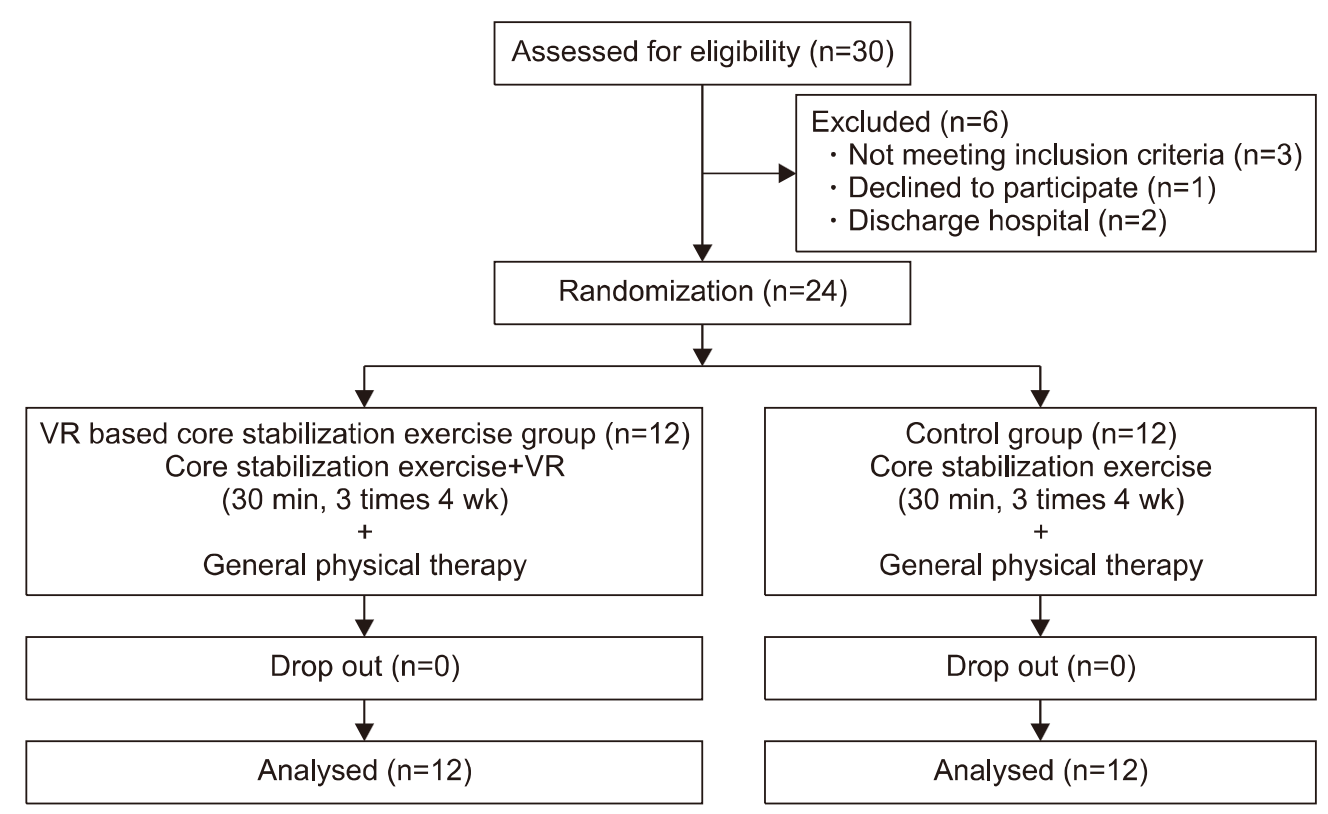

Figure 1. Schematic of the design of the study. VR: virtual reality. 
ceived general physical therapy for 30 minutes, 3 times a week for 4 weeks, and the experimental group conducted VR-based trunk-centered stabilization exercise for $30 \mathrm{mi}$ nutes, 3 times a week for 4 weeks. Trained physiotherapists facilitated the programs as they are highly familiar with the types of exercise and the possible side effects, and the same therapist conducted the program (Figure 1).

\section{Experimental methods}

\section{Virtual reality-based core stabilization exercise group}

The experimental group performed VR-based stabilization exercise 3 times a week for 4 weeks, and the stabilization exercise of Urquhart et al. [12], which comprised 5 minutes of warm-up exercise, 20 minutes of main exercise, and 5 minutes of cool down exercise, was also performed. In total, eight exercise programs, which comprised 5 minutes of warm-up exercise, 20 minutes of main exercise, and $5 \mathrm{mi}$ nutes of cool down exercise, were performed in the VR environment (Table 1).

In the VR-based core stabilization exercise, a sunglasstype VR device (HMD, VR EYES; SMART PIA, Yongin, Korea, 2017), notebook (LG14Z96; LG Electronics, Seoul, Korea, 2016), smartphone (A1586; Apple, Cupertino, CA,
USA), and program that converts a normal video screen into a VR (Homido 360 player; HMD-TECH, Lille, France, 2015) were used (Figure 2).

The pre-recorded video with a resolution of $1,280 \times 720$ was connected to a VR environment and was attached to the VR device for viewing. The participants were instructed to perform the exercise according to the exercise image (Figure 3).

When a patient did not accurately execute the exercise for more than 3 times, the therapist provided a signal to facilitate the correct movement. In the cool down exercise, the pressure was set at $30 \mathrm{mmHg}$ using a pressure biofeedback device (Chattanooga Stabilizer Pressure Biofeedback; Chattanooga Group, Hixson, TN, USA, 2011), which was composed of an air bag and a pressure gauge, to maintain a constant pressure in the abdomen.

After performing one program 6 times, the participants took a 1-minute rest to minimize physical fatigue. The exercise was performed 3 times a week for 4 weeks. If some of the exercises could not be performed by the patients themselves during the training process, a research staff personnel assisted them.

Table 1. Contents of the core stabilization exercise program

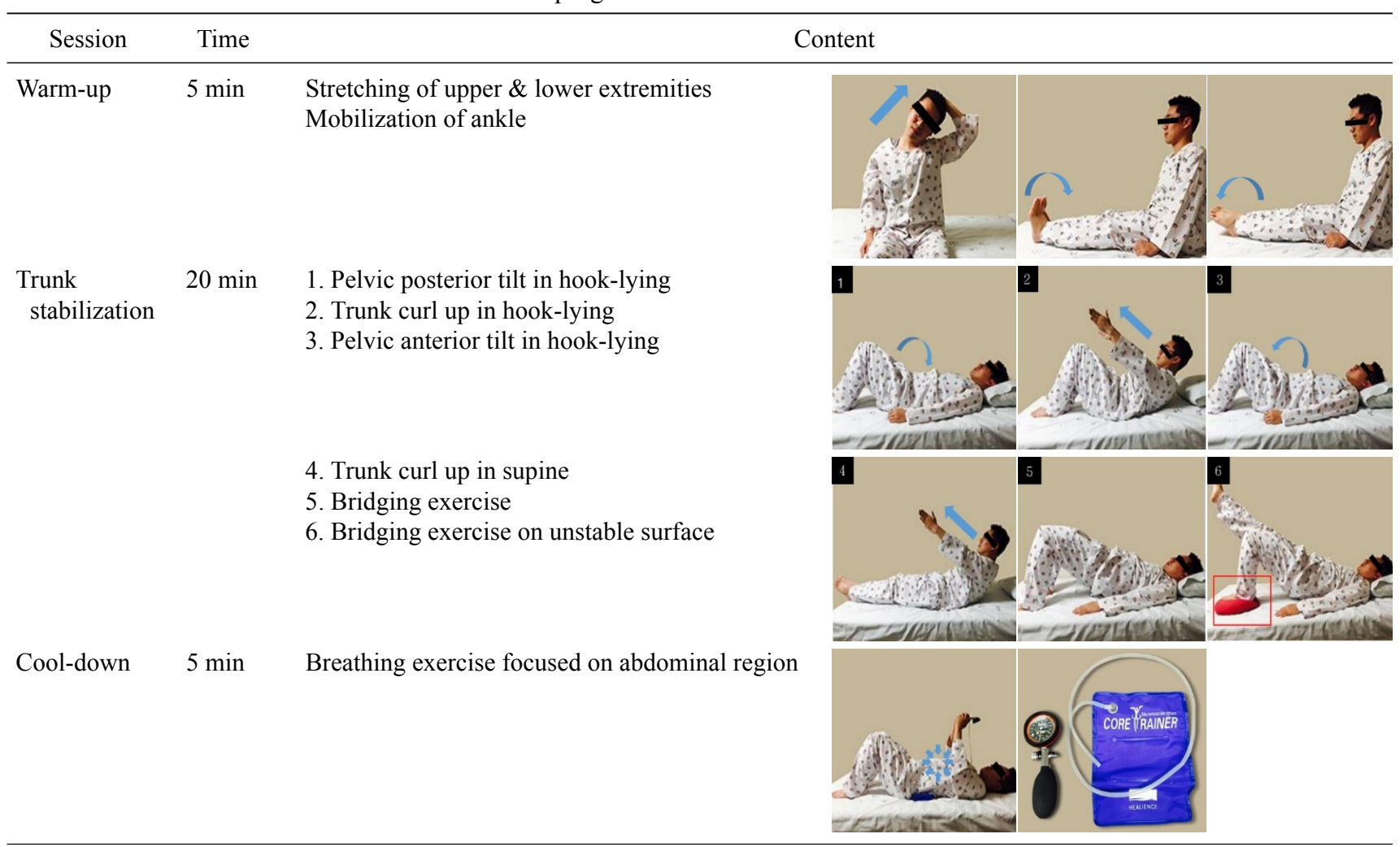




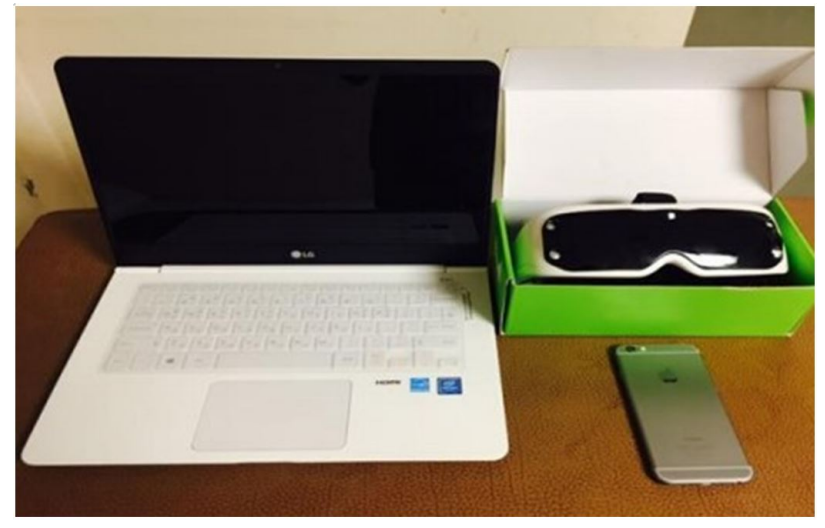

Figure 2. Virtual reality device.

\section{General core stabilization exercise program}

The participants performed the trunk movement exercise according to the non-VR-based trunk center stabilization exercise program. Eight movements were performed in the same manner as in the VR-based trunk exercise, and after each movement was performed 6 times, the participants took a 1-minute break to minimize fatigue. The exercise was performed for 30 minutes, 3 times a week for 4 weeks.

\section{General physical therapy}

General physical therapy is a central nervous system development treatment performed as a 1:1 session with a physical therapist. Both groups received general physical therapy for 30 minutes, 5 times a week for 4 weeks according to the treatment plan. The exercise programs included stretching, joint movement, and strength training.

\section{Measurement tools}

\section{Manual functional test}

The manual function test (MFT) comprises task for upper limb movement (4 items), grasp ( 2 items), and finger manipulation ( 2 items). In this test, the participants could score a maximum of 32 points, and a higher score indicated better upper extremity function. The intra-rater reliability is 0.95 , and the inter-rater reliability is 0.95 [13].

\section{Box and block test}

The box and block test (BBT) was used to investigate changes in upper extremity function after treatment. This test was performed by counting the number of blocks, measuring 1 inch, that were moved from one box to another within 1 minute. The participants were provided with a 15 -s practice session and the values were measured afterwards. The

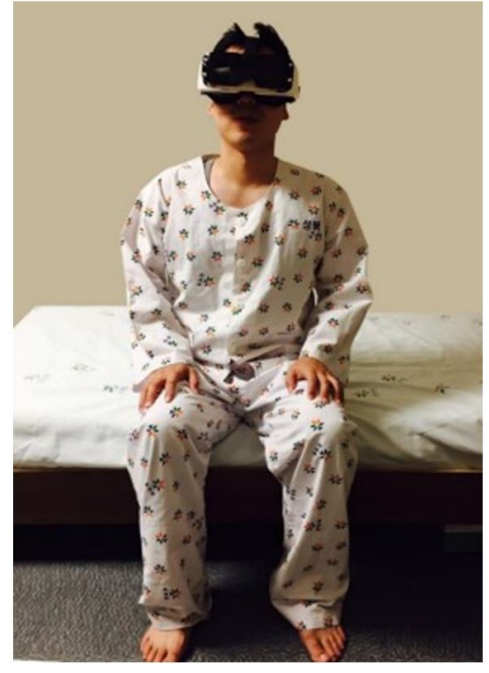

Figure 3. Application of virtual reality training.

intra-rater reliability was 0.94 , and the inter-rater reliability was 0.99 [14].

After converting the data into digital signals they were wirelessly sent to a personal computer via BioScan (BIOBRAIN, Daejeon, Korea, 2017) which is a professional electromyogram analysis program. The Bioscan was applied with a 4-500 HZ band-pass filter. After applying 60, 120, 180 HZ Notch Filter to remove noise of the power supply, the RMS index, which reflects muscle activation, was calculated. The BBS, postural assessment scale for stroke, and the Korean version of the geriatric depression scale (K-TIS) were used to measure changes in postural control after the intervention. The BBS is designed to evaluate static and dynamic balance of patients with nervous system disorders. Moreover, it is composed of 14 items and is categorized into three: sitting, standing, and posture change. In this test, the maximum total score is 56 points. The intra-rater reliability is 0.99 , and the inter-rater reliability is $0.98[15,16]$.

The K-TIS was used to evaluate the trunk movement of the participants with stroke and to measure static balance, dynamic balance, and trunk coordination while in a sitting position. It comprised three items for static balance and 10 items for dynamic balance while in sitting position, and the score can range from 0 to 23 . The inter-rater reliability ranged from 0.87 to 0.96 , and the inter-rater reliability ranged from 0.85 to 0.99 [17].

The geriatric depression scale (GDS) was used to measure changes in depression after the intervention. This is a dichotomous scale with 30 questions that can be answered with a 
yes/no answer. A higher score indicated a worse state of depression. A total score of 5-15 indicated normal mood; 6-9 points indicated moderate depression; and 10 points or more indicated depression. The test-retest reliability was as high as 0.85 [18].

\section{Statistical analysis}

For the analysis and statistical processing of data in this study, the mean and standard deviation were calculated using the SPSS for Windows Version 21.0 (IBM Co., Armonk, NY, USA). All participants were tested for normality, and the general characteristics of the participants were assessed using descriptive statistics. A paired t-test was performed to compare the scores of the experimental and control groups before and after the intervention. An independent t-test was conducted to investigate the differences between the two groups according to intervention. The significance level was set at 0.05 .

\section{Results}

\section{General characteristics of subject}

The average age of the VR + trunk stability group was 71.92, and the control group was 72.08. In the VR+trunk stability group, there were 5 male and 7 female, and the control group included 2 male and 10 female. The period after onset was found to be 17.91 months in the VR + trunk stability group and 18.75 months in the control group (Table 2).
Changes in functional ability in the upper extremity according to interventions

The MFT score of the VR + trunk stability group showed a significant difference from 14.50 points to 17.31 points, and the control group also showed a significant difference from 15.00 points to 17.16 points. The comparison between groups showed a significant difference in the VR + trunk stability group. In the BBT test, the VR + trunk stability group showed a significant difference from 2.08 to 3.83 , and the control increased from 2.08 to 2.75 . The comparison between groups showed a more significant difference in the VR + trunk stability group (Table 3 ).

\section{Changes in postural control according to the intervention}

For the BBS scores, the VR + trunk stability group increased from 32.58 points to 37.58 points, and the control group increased from 33.50 points to 35.75 points. The comparison between groups showed a more significant difference in the VR + trunk stability group. In the K-TIS, the VR-based core stabilization exercise showed a significant difference from 13.75 points to 15.41 , and the control group increased from 11.00 points to 11.91 points. In the comparison between groups, the VR + trunk stability group showed a more significant difference (Table 4).

\section{Changes in depression according to the intervention}

In the K-GDS, the VR + trunk stability group showed a significant difference from 16.00 to 12.16 , and the control group showed a significant difference from 17.08 to 12.75 (Table 5).

Table 2. General characteristics of subject

\begin{tabular}{lcrr}
\hline \multicolumn{1}{c}{ Parameters } & Virtual reality-based core stabilization group $(\mathrm{n}=12)$ & Control group $(\mathrm{n}=12)$ & $\mathrm{t} / \chi^{2}(p)$ \\
\hline Age $(\mathrm{y})$ & $71.92(3.23)$ & $72.08(4.46)$ & $0.015(0.917)$ \\
Sex $(\mathrm{n})$ & & & \\
Male & $5(41.7)$ & $2(16.7)$ & $1.342(0.194)$ \\
Female & $7(58.3)$ & $10(83.3)$ & $-1.341(0.200)$ \\
Height (cm) & $161.08(6.94)$ & $158.16(2.91)$ & $0.043(0.966)$ \\
Weight (kg) & $65.08(8.68)$ & $64.91(10.04)$ & $-3.940(0.698)$ \\
Lesion site (left/right) & $6 / 6$ & $5 / 7$ & $0.793(0.436)$ \\
Cause of onset & $5 / 7$ & $2 / 10$ & \\
(hemorrhage/infarction) & & & $18.75(3.81)$ \\
Duration after onset (mo) & $17.91(3.20)$ & $26.58(1.08)$ & $-1.704(0.103)$ \\
K-MMSE (score) & $27.33(1.07)$ & & \\
\hline
\end{tabular}

Values are presented as mean (SD), number (\%), or number only. K-MMSE: Korea mini-mental state examination. 
Table 3. Changes in functional ability in the upper extremity according to interventions

\begin{tabular}{lccc}
\hline \multicolumn{1}{c}{ Parameters } & Virtual reality-based core stabilization group $(\mathrm{n}=12)$ & Control group $(\mathrm{n}=12)$ & $\mathrm{t}(p)$ \\
\hline MFT (score) & & & $1.106(0.281)$ \\
Pre-test & $14.50(1.24)$ & $15.00(0.95)$ & $2.16(1.11)$ \\
Post-test & $17.31(1.16)$ & $2.16(1.26)$ & $2.100(0.047)$ \\
Change value & $3.41(1.62)$ & $5.920(<0.001)$ & \\
$\mathrm{t}(p)$ & $7.30(<0.001)$ & & \\
BBT (score) & & $2.08(0.90)$ & $0.000(1.000)$ \\
Pre-test & $2.08(0.79)$ & $2.75(0.96)$ & \\
Post-test & $3.83(1.11)$ & $0.66(0.88)$ & $2.330(0.029)$ \\
Change value & $1.75(0.96)$ & $2.600(0.025)$ & \\
$\mathrm{t}(p)$ & $6.280(<0.001)$ & & \\
\hline
\end{tabular}

Values are presented as mean (SD).

MFT: manual functional test, BBT: box and block test.

Table 4. Changes in postural control according to the intervention

\begin{tabular}{lccc}
\hline \multicolumn{1}{c}{ Parameters } & Virtual reality-based core stabilization group $(\mathrm{n}=12)$ & Control group $(\mathrm{n}=12)$ & $\mathrm{t}(p)$ \\
\hline BBS (score) & & & \\
Pre-test & $32.58(6.45)$ & $33.50(4.66)$ & \\
Post-test & $37.58(5.64)$ & $35.75(4.61)$ & $2.160(0.041)$ \\
Change value & $5.00(2.69)$ & $2.25(3.46)$ & \\
$\mathrm{t}(p)$ & $6.420(<0.001)$ & & \\
$\mathrm{K}$-TIS (score) & & & \\
Pre-test & $13.75(1.48)$ & $11.00(1.47)$ & $1.91(1.44)$ \\
Post-test & $15.41(1.24)$ & $0.91(1.16)$ & $1.290(0.207)$ \\
Change value & $1.66(1.77)$ & $2.720(0.020)$ & \\
$\mathrm{t}(p)$ & $3.250(0.008)$ & & \\
\hline
\end{tabular}

Values are presented as mean (SD).

BBS: Berg balance scale, K-TIS:Korea trunk impairment scale.

Table 5. Changes in depression according to the intervention

\begin{tabular}{lccc}
\hline \multicolumn{1}{c}{ Parameters } & Virtual reality-based core stabilization group $(\mathrm{n}=12)$ & Control group $(\mathrm{n}=12)$ & $\mathrm{t}(\mathrm{p})$ \\
\hline K-GDS (score) & & & \\
Pre-test & $16.00(1.04)$ & $17.08(1.37)$ & \\
Post-test & $12.16(0.71)$ & $-4.33(1.15)$ & $-0.920(0.364)$ \\
Change value & $-3.83(1.46)$ & $-13.000(<0.001)$ & \\
$\mathrm{t}(p)$ & $-9.050(<0.001)$ & &
\end{tabular}

Values are presented as mean (SD).

K-GDS: Korea geriatric depression scale.

\section{Discussion}

The MFT is a useful tool for the evaluation of upper limb function. That is, it can easily reflect the recovery of upper limb function to a practical level, which is associated with activities of daily living, and it can be objectively implemented. In this study, the MFT test was performed to investigate changes in the upper and lower extremity functions of the
VR-based core stabilization exercise group. The score of the VR-based exercise group increased from 14.50 to 17.91 $(p<0.05)$. In this study, the muscles used for core stabilization exercise were postural muscles, which play an important role in stability and postural control of the trunk during whole-body exercises and provides stability to promote smooth limb movements. In relation to this, the upper limb function also improved due to increased trunk stability, and 
the range of motion significantly increased due to the utilization of large muscles, which are easy to use, at a higher frequency. In addition, VR training promotes the reorganization of the cerebral cortex into neurological movements. Hence, repetitive exercises can improve weakened trunk muscles. In addition, the VR-based exercise program promotes patient interest and active participation compared to traditional treatment methods conducted by therapists, and it increases repetition, intensity, and task-based learning and improves the function of trunk-based muscles.

In this study, improvement in the BBT score indicates better overall manipulation function and upper limb movement. Considering that the average age of the participants was high, only a few blocks were used in the pre-evaluation, and results showed low hand activity with insufficient use. However, the result was considered significant due to the remarkable difference after the intervention in this study.

These results can be supported by previous studies showing that trunk stability improved with the VR-based program consisting of core stabilization exercise. Moreover, these studies revealed that upper limb movement resulted in a balanced activity between the cerebral hemispheres [19] Thus, the improvement of upper extremity function was attributed to better trunk stability; with this improvement, the interest and concentration levels of the participants increased. In addition, repetitive training is an important factor in efficiently increasing exercise learning.

The VR-based exercise program used in this study emphasized in real time how to accurately perform the movements, and the therapist provided immediate feedback in regards to incorrect performance. These training conditions led to an increase in task-related feedback, which is the most important element of exercise learning, and the effect improved with the increased amount of exercise learning. In the process of functional recovery after stroke, patients first present with improvement in trunk stabilization, followed by improved upper limb function, which is important for activities of daily living [20].

That is, the trunk function of stroke patients can improve with core stabilization training, and patients can experience improved upper limb function while in a stable position. In turn, VR-based training can motivate and satisfy patients, and upper extremity function can increase with improved pelvic and trunk stability, which is required for effective mobilization of the upper limb [6].

Previous studies by Walker et al. [21] showed that after the application of a VR-based exercise program, the BBS score of stroke patients increased from 43.8 to 48.8 . In this study, the BBS score of the VR-based core stabilization exercise group improved significantly from 32.58 to 40.25 , which was consistent with the results of previous studies $(p<0.05)$.

In addition, a significant difference was observed before and after the experiment in the control group, and this result showed that not only was the VR-based trunk-based exercise but also general core stabilization exercise are effective in improving balance among patients with stroke. These patients lose their balance due to impaired coordination and proprioception function caused by damage to the central nervous system [22].

This study provided a realistic VR-based exercise to the patient, which projects the stabilization movement of the body. After movement-related sensory information introduced through VR is integrated in the central nervous system, posture control ability and balance will improve by activating body movements via interactions in the cerebral region (motor cortex and sensory cortex), which is correlated to motor output.

A study by Hodges and Richardson [23] showed that spine stabilization due to the contraction of the abdominal and multifidus muscles, which are active prior to the movement of the limbs, must be regulated in the central nervous system. Therefore, in this study, core stabilization exercises, which strengthen the abdominal and multifidus muscles, was effective in inducing the activity of the central nervous system necessary for posture control. Moreover, the K-TIS score of the VR-based core stabilization exercise group increased from 13.75 to 15.41 before the experiment $(p<0.05)$, and the score of the control group also significantly increased from 11.00 to 11.91 . Thus, a significant difference was observed $(p<0.05)$. However, there was no significant difference between the two groups because the homogeneity of the K-TIS score was not confirmed in this study. Thus, whether the comparison of the training effect is valid must be considered. In this study, the participants find it difficult to assume the position required for K-TIS examination. Thus, several items were assigned a score of 0 , thereby showing that the treatment had a minimal effect. In addition, considering that the experimental period of the preceding study was 6 or 8 weeks, a difference was not observed between the groups due to the difference in the intervention period. Therefore, these exercise programs and virtual trunk-based stabilization exercises are effective in improving posture control in patients with stroke survivors. 
More than $50 \%$ of patients with stroke present with depression because they are isolated from family, and their activities of daily living are affected due to the loss of independence in performing these activities. Therefore, mental problems, such as depression, apathy, and anxiety, should be considered in the recovery process. Depression can be reduced through the improvement of physical activity in the recovery process in stroke patients [24]. In this study, to improve depression, a VR program-based exercise program was applied to show the significant differences before and after the experiment in each group $(p<0.05)$. However, no significant difference was observed between the two groups. This result may be attributed to the fact that most participants had were affected with chronic stroke. Thus, their expectations about hospital life and improvement in physical function were relatively low.

Kivela et al. [25] claimed that there were differences in the pattern of depression between male and female. That is, male were more likely to experience insomnia and decreased interest and stagnation in the early and middle stages. Meanwhile, female experienced a higher level of anxiety, more physical symptoms, and early insomnia [25]. The participants in this study comprised mainly older female. In this study, there was no significant change in depression after the intervention.

The control group also showed a significant difference, but in the comparison of the effect difference between groups, the score change in the VR-based core stabilization exercise group was found to be more significant. These results are thought to show the therapeutic effect of the general core stabilization exercise and the relative superiority of the VR-based core stabilization exercise at the same time. Although there is concern about the discomfort caused by wearing the VR device, none of the participants complained of the discomfort of wearing it. Rather, it was familiar with wearing sunglasses, so the side effects of the application method were not considered.

The current study had several limitations. That is, the results of the current study cannot be generalized due to the small number of participants, and the intervention period was short. Since this study only included a few participants who met the inclusion criteria, the results cannot be generalized to all stroke patients. Lastly, sufficient data about the maintenance of treatment effect cannot be obtained because follow-up was not conducted.

The VR-based core stabilization exercise was effective in improving upper limb function and posture control, and the use of K-TIS and K-GDS must be assessed in future studies. Moreover, the VR-based programs are suitable for patients with stroke. However, further studies with a long intervention period should be conducted to assess the effects of training programs on the recovery of stroke patients. Thus, the VR-based training in rehabilitation has a potential value for stroke patients, and the application of this method in clinical trials must be diversified.

\section{Conflict of Interest}

The authors declared no potential conflicts of interest with respect to the authorship and/or publication of this article.

\section{References}

1. Carr JH, Shepherd RB, Nordholm L, Lynne D. Investigation of a new motor assessment scale for stroke patients. Phys Ther 1985; 65:175-80.

2. Kim JC, Lee HM. The effect of action observation training on sit to walk with chronic stroke patients. J Korean Phys Ther 2015: 27:413-8

3. Sackley CM. Falls, sway, and symmetry of weight-bearing after stroke. Int Disabil Stud 1991;13:1-4.

4. Hsieh CL, Sheu CF, Hsueh IP, Wang CH. Trunk control as an early predictor of comprehensive activities of daily living function in stroke patients. Stroke 2002;33:2626-30.

5. Shin JH, Kim MY, Lee JY, Jeon YJ, Kim S, Lee S, et al. Effects of virtual reality-based rehabilitation on distal upper extremity function and health-related quality of life: a single-blinded, randomized controlled trial. J Neuroeng Rehabil 2016;13:17.

6. Lee MM, Shin DC, Song CH. Canoe game-based virtual reality training to improve trunk postural stability, balance, and upper limb motor function in subacute stroke patients: a randomized controlled pilot study. J Phys Ther Sci 2016;28:2019-24.

7. Cho K. The effects of virtual reality based treadmill training on the muscle architecture of gastrocnemius in chronic stroke patients: randomized controlled trial. J Korean Phys Ther Sci 2017; 24:1-11.

8. Roh JS. The effect of virtual reality based rehabilitation program on balance of patient with stroke: a meta-analysis of studies in Korea. J Korean Phys Ther Sci 2017;24:59-68.

9. Van Schaik P, Blake J, Pernet F, Spears I, Fencott C. Virtual augmented exercise gaming for older adults. Cyberpsychol Behav 2008;11:103-6.

10. Zelinski EM, Reyes R. Cognitive benefits of computer games for older adults. Gerontechnology 2009;8:220-35.

11. Masiero S, Celia A, Rosati G, Armani M. Robotic-assisted rehabilitation of the upper limb after acute stroke. Arch Phys Med Rehabil 2007;88:142-9.

12. Urquhart DM, Hodges PW, Allen TJ, Story IH. Abdominal muscle recruitment during a range of voluntary exercises. Man Ther $2005 ; 10: 144-53$. 
13. Miyamoto S, Kondo T, Suzukamo Y, Michimata A, Izumi S. Reliability and validity of the Manual Function Test in patients with stroke. Am J Phys Med Rehabil 2009;88:247-55.

14. Mathiowetz V, Volland G, Kashman N, Weber K. Adult norms for the Box and Block Test of manual dexterity. Am J Occup Ther 1985;39:386-91.

15. Bogle Thorbahn LD, Newton RA. Use of the Berg Balance Test to predict falls in elderly persons. Phys Ther 1996;76:576-83; discussion 584-5.

16. Kim SC, Hur YG. The effect of treadmill and body weight support treadmill training on balance and gait ability in hemiplegia patients. J Korean Phys Ther Sci 2018;25:31-43.

17. Verheyden G, Nieuwboer A, Van de Winckel A, De Weerdt W. Clinical tools to measure trunk performance after stroke: a systematic review of the literature. Clin Rehabil 2007;21:387-94.

18. Yesavage JA, Brink TL, Rose TL, Lum O, Huang V, Adey M, et al. Development and validation of a geriatric depression screening scale: a preliminary report. J Psychiatr Res 1982-1983;17: $37-49$.

19. Cauraugh JH, Kim SB, Duley A. Coupled bilateral movements and active neuromuscular stimulation: intralimb transfer evidence during bimanual aiming. Neurosci Lett 2005;382:39-44.
20. Verheyden G, Vereeck L, Truijen S, Troch M, Herregodts I, Lafosse $\mathrm{C}$, et al. Trunk performance after stroke and the relationship with balance, gait and functional ability. Clin Rehabil 2006; 20:451-8.

21. Walker ML, Ringleb SI, Maihafer GC, Walker R, Crouch JR, Van Lunen B, et al. Virtual reality-enhanced partial body weight-supported treadmill training poststroke: feasibility and effectiveness in 6 subjects. Arch Phys Med Rehabil 2010;91:115-22.

22. Smania N, Picelli A, Gandolfi M, Fiaschi A, Tinazzi M. Rehabilitation of sensorimotor integration deficits in balance impairment of patients with stroke hemiparesis: a before/after pilot study. Neurol Sci 2008;29:313-9.

23. Hodges PW, Richardson CA. Altered trunk muscle recruitment in people with low back pain with upper limb movement at different speeds. Arch Phys Med Rehabil 1999;80:1005-12.

24. Mather AS, Rodriguez C, Guthrie MF, McHarg AM, Reid IC, McMurdo ME. Effects of exercise on depressive symptoms in older adults with poorly responsive depressive disorder: randomised controlled trial. Br J Psychiatry 2002;180:411-5.

25. Kivelä SL, Pahkala K, Laippala P. Prevalence of depression in an elderly population in Finland. Acta Psychiatr Scand 1988;78: 401-13. 